\title{
1 Phylogenetic annotation and genomic architecture of opsin genes in
}

2 Crustacea

4 Jorge L. Pérez-Moreno*1, Danielle M. DeLeo*1, Ferran Palero², Heather D. Bracken5 Grissom $^{1}$

$6{ }^{1}$ Department of Biological Sciences, Florida International University - Biscayne Bay Campus, $7 \quad$ North Miami FL 33181, U.S.A.

$8{ }^{2}$ Centre d'Estudis Avançats de Blanes (CEAB-CSIC), Carrer d'Accés a la Cala Sant Francesc 9 14, 17300 Blanes, Spain.

11 * Equally contributing authors

13 Corresponding author: Jorge L. Pérez-Moreno jorge.perezmoreno@fiu.edu +17862130026

\section{Abstract}

A major goal of evolutionary biology is to understand the role of adaptive processes on sensory systems. Visual capabilities are strongly influenced by environmental and ecological conditions, and the evolutionary advantages of vision are manifest by its complexity and ubiquity throughout Metazoa. Crustaceans occupy a vast array of habitats and ecological niches, and are thus ideal taxa to investigate the evolution of visual systems. A comparative approach is taken here for efficient identification and classification of opsin genes, photoreceptive pigment proteins involved in color vision, focusing on two crustacean model organisms (Hyalella azteca and Daphnia pulex). Transcriptomes of both species were assembled de novo to elucidate the diversity and function of expressed opsins within a robust phylogenetic context. For this purpose, we developed a modified version of the Phylogenetically Informed Annotation tool to filter

31 and identify visual genes from transcriptomes in a scalable and efficient manner. 
Additionally, reference genomes of these species were used to validate our pipeline while characterizing the genomic architecture of the opsin genes. Next-generation sequencing and phylogenetics provide future venues for the study of sensory systems, adaptation, and evolution in model and non-model organisms.

Keywords: evolution; phototransduction; protein; rnaseq; transcriptomics; vision

\section{Introduction}

Opsins are photoreceptor molecules that play a crucial role in animal vision and can be found across metazoans (Terakita, 2005). As membrane-associated, G-proteincoupled receptors (GPCRs), opsins can function in both visual and non-visual phototransduction, and in some instances as photoisomerases (Shichida \& Matsuyama, 2009). Previous studies have classified opsins in three primary categories according to the type of G-protein to which they couple namely, "ciliary" (c-opsins), "rhabdomeric" ( $r$ opsins), and RGR/Go opsins (Terakita, 2005; Feuda et al., 2014, 2016). Ciliary and rhabdomeric opsins diversified prior to the protostome-deuterostome split and are found in both invertebrates and vertebrates, which suggests that they co-occurred in a common ancestor (Kojima et al., 1997; Shichida \& Matsuyama, 2009; Hering \& Mayer, 2014; Ramirez et al., 2016). Opsin categories can additionally be further subdivided into subfamilies based on molecular phylogenetics and functional classifications (Terakita, 2005). These subfamilies share less than 20 percent amino acid identity (Fryxel \& Meyerowitz, 1991) and comprise c-opsins (visual and non-visual); tmt/encephalopsins; Go- and Gs- coupled, (r-opsins) Gq-coupled/melanopsins; and photoisomerases/ neuropsins. Photoreceptive opsins can be of either the ciliary-type, found largely in vertebrates (for exceptions see Arendt et al., 2004; Passamaneck et al., 2011; Bok et al., 2017; Tsukamoto et al., 2017), or the rhabdomeric-type found in mollusks, annelids, and the compound eyes of arthropods (Arendt et al., 2002; Shichida \& Matsuyama, 2009; Gühmann et al., 2015), with the last being the focus of the present study. 
Opsins form visual pigments capable of absorbing photons when bound to a chromophore, generally a vitamin A1 derivative (11-cis retinal). These visual pigments trigger conformational changes that activate G-proteins (Nathans, 1987) and elicit phototransduction signaling cascades. Key biological processes such as the regulation of circadian clocks, phototaxis, and vision have been shown to be linked to the phototransduction cascade (e.g. Arendt et al., 2004; review Shichida \& Matsuyama, 2009). The set of amino acid residues that interact with the chromophore produce an environment suitable for the absorption of light with distinct wavelengths (Imai et al., 1997; Kuwayama et al., 2002) and thus influence spectral tuning (e.g. Porter et al., 2007; Katti et al., 2010). As the absorption spectrum of the photopigment is influenced by the amino acid composition of the opsin protein, slight variations can alter its physical and chemical properties and lead to visual pigments maximally sensitive to different wavelengths of light. This in turn would allow organisms to perceive and distinguish between light of particular wavelengths. The direct association between amino acid composition of photoreceptive opsins and their spectral sensitivity make them amenable to functional classification by sequence analysis (Mirzadegan et al., 2003; Matsumoto \& Ishibashi, 2016).

Three main approaches have been employed to characterize opsins from transcriptomic data: I) Sequence similarity searches via pairwise alignments (i.e. BLAST); II) Protein structure prediction through Hidden Markov Model (HMM) profile sequence similarity is typically based on heuristic algorithms that search for matching nucleotide and/or amino acid sequences in curated databases (e.g. BLAST; Altschul et al., 1990). Sequences are locally or globally aligned and subsequently annotated based on inferred homology with statistically significant matches (Pearson, 2013). These comparisons, however, can rapidly become computationally expensive as the number of query and/or reference sequences increases (Suzuki et al., 2012). Although similarity searches via pairwise alignments are capable of identifying homologous sequences,

92 their shortcomings are notorious when the queries consist of protein families with low sequence similarities, as it is the case for opsins and other GPCRs (Pearson, 2013). 
94 Hidden Markov Model (HMM) methods offer an enticing alternative to pairwise 95 alignments at similar computational costs (Eddy, 2011; Pearson, 2013). HMM profiles can also contain relevant information regarding protein structure, which translates to more accurate identification, classification, and annotation of proteins even when overall sequence similarity is low (Krogh et al., 1994; Yoon, 2009; Pearson, 2013). However, the efficacy of HMMs is intrinsically dependent on the quality of the training data, which is a non-trivial process in the case of understudied taxa or protein families (Rasmussen \& Krink, 2003; Pearson, 2013). Therefore, the use of HMMs for annotation of GPCRs is hindered when independently verified sequences are not readily available. The robustness and suitability of phylogenetic approaches for functional annotation of opsins

104 (and other proteins) is unparalleled, as it can readily overcome many of the deficiencies 105 of other homology-based methodologies (Engelhardt et al., 2009; Gaudet et al., 2011; 106 Speiser et al., 2014). The placement of proteins on a phylogenetic tree not only enables 107 a rapid assessment of homology and efficient discrimination of false-positives, but also 108 allows for the inference of putative functions and roles within an evolutionary context 109 (Engelhardt et al., 2009). This approach has been successful in classifying novel opsins 110 (and other GPCRs) despite their characteristic low sequence similarities and, in the 111 case of non-model organisms, scarce genomic resources (Porter et al., 2007, 2012; 112 Speiser et al., 2014). The main drawbacks of phylogenetic reconstruction as an efficient 113 functional annotation method are possible difficulties aligning distantly related 114 sequences, its propensity to be time-consuming (obtaining adequate references, computation of trees, etc.) and the steep learning curve to master these analyses, which 116 might result in subjectivity and misinterpretations (Crisp \& Cook, 2005).

Efforts to characterize opsins from high-throughput sequencing data in non119 model Crustacea have primarily focused on transcriptomes, but without genomic 120 validation (Porter et al., 2013; Wong et al., 2015; Biscontin et al., 2016). When 121 available, genomes can provide valuable information regarding opsin gene duplication 122 in an organism, as well as the relative locations of those genes. Gene locations allow for 123 intra- and interspecific comparisons (Nordström et al., 2004) and to make inferences 
124 about the evolutionary history of opsin diversification (review Shichida \& Matsuyama, 125 2009).

126 In this study we modified the Phylogenetically Informed Annotation tool's pipeline 127 (Speiser et al., 2014) to conduct a robust and scalable phylogenetic annotation of visual 128 opsins from transcriptomes of two crustacean model organisms, Hyalella azteca and 129 Daphnia pulex. Hyalella azteca is a freshwater epibenthic amphipod, commonly used as 130 a bioindicator species, which has one pair of pigmented compound eyes (Gonzalez \& 131 Watling, 2002). Daphnia pulex is a freshwater cladoceran that has a single but movable 132 cyclopean, compound, and pigmented eye. Specifically, we made modifications for PIA 133 to run on the command-line rather than on Galaxy's GUI and wrote wrapper scripts to 134 facilitate the analyses. This resulted in a scalable and automated platform to annotate 135 visual genes and pathways, while minimizing possible biases and subjectivity from 136 manual curation. As genomes are available for both species, they were used to validate 137 the annotations and make inferences about the genomic architecture and the opsin 138 intron-exon gene structure within these species.

\section{Methods}

Data, Quality Control, and Transcriptome Assembly

Raw RNA sequencing data of the freshwater amphipod Hyalella azteca and the model branchiopod Daphnia pulex were downloaded from the NCBI's Sequence Read Archive (SRA). In order to facilitate de novo transcriptome assembly and accurate detection of complete opsin isoforms, the read files were trimmed taking into consideration factors such as length and quality of the sequencing reads, sequencing

150 Table 1. Raw data chosen for de novo transcriptome assembly and annotation of opsin proteins in 151 Hyalella azteca and Daphnia pulex.

\begin{tabular}{c|cccccc} 
Species & Megabytes & Megabases & Read lengths & $\begin{array}{c}\text { Sequencing } \\
\text { Platform }\end{array}$ & Tissue type & SRA BioProject \\
\hline Hyalella azteca & 15543 & 33160 & $2 \times 150 \mathrm{bp}$ & Illumina HiSeq & Whole & PRJNA312414
\end{tabular}


152

153

154

155

Prior to the assembly process, quality of the raw sequencing reads was evaluated via FastQC (Andrews, 2010). The FastQC output was subsequently used to inform stringent quality and adaptor trimming with Trimmomatic 0.36 (parameters: "ILLUMINACLIP:TruSeq3-PE.fa:2:30:10 CROP:140 HEADCROP:20 LEADING:15 TRAILING:15 SLIDINGWINDOW:4:20 MINLEN:36"; Bolger et al., 2014). Clean sequencing reads were then assembled into a de novo transcriptome with the Trinity pipeline (version 2.4.0; Grabherr et al., 2011; Haas et al., 2013) using default parameters, a minimum contig length of 200bp, and a kmer size of 23. Assembly summary statistics were calculated using Transrate 1.0.3 (Smith-Unna et al., 2016). BUSCO 3.0.2 (Benchmarking Universal Single-Copy Orthologs; (Simão et al., 2015) was employed to assess the quality and completeness of the resulting transcriptomes. The latter method provides an accurate evaluation of transcriptomes in an evolutionary informed context by assessing the presence and completeness of universal single copy orthologs (Simão et al., 2015). BUSCO analyses were conducted with the Arthropoda database of orthologous groups $(n=1066)$ sourced from OrthoDB (Waterhouse et al., 2013).

\section{Identification and Annotation of Crustacean Opsins}

Identification and functional classification of putative opsin transcripts was achieved through the use of our modified version of the existing PhylogeneticallyInformed Annotation (PIA) tool (Speiser et al., 2014). While phylogenetic confirmation of BLAST similarity hits is becoming routine in model systems, PIA allows for the identification of proteins involved in visual pathways for non-model organisms in a computationally efficient manner (Speiser et al., 2014). This informative tool places putative visual gene transcripts (e.g. opsins), previously identified via BLAST searches against a custom database, in pre-computed phylogenies of such genes. The resulting phylogenies can then be used to discriminate BLAST false-positives and/or paralogous sequences from the transcripts of interest. While PIA has been used in previous studies 
182 to annotate genes in a phylogenetic context, it was originally designed as a workflow for 183 the Galaxy Project (Afgan et al., 2016) and as such is dependent on a Graphical User 184 Interface (Speiser et al., 2014). This workflow can become inefficient when conducting 185 concurrent analyses of numerous transcriptomes. Further, curation of the phylogenetic 186 gene trees produced by PIA for each input transcriptome is typically undertaken 187 manually, which inevitably makes it sensitive to potential biases. Tree branch length 188 cutoff values for a given gene (i.e. opsins) can however be determined empirically 189 through a series of manual tree curation comparisons. The pipeline presented here is a 190 modification to PIA's source code in which the authors wrote a wrapper script to enable 191 its use as a command-line/automated workflow, which effectively increases its 192 scalability allowing for the identification of visual opsins from multiple transcriptomic 193 datasets through simple scripting. Although the pipeline was designed for analyses of 194 visual pathways, it is possible to create custom databases and phylogenies for 195 customized analyses of other genes/pathways. We refer the reader to the original 196 publication of PIA for additional information regarding included genes and pathways 197 (Speiser et al., 2014). The modified Phylogenetically Informed Annotation pipeline 198 employed in this study, along with usage examples, will be made available at: 199 http://xibalbanus.github.io scanned with Biopython's get_orfs_cds.py script (Cock et al., 2009) to translate each transcript into its corresponding amino acid sequence. Open Reading Frames (ORFs) were then extracted via the same script to facilitate the PIA annotation process. After 205 conclusion of PIA's main component (BLAST, MAFFT alignment, and phylogenetic placement via RAxML; Altschul et al., 1990; Stamatakis, 2014; Yamada et al., 2016) , a script adapted from the Osiris Phylogenetics toolkit (long_branch_finder.py; Oakley et 208 al., 2014) was used to identify transcripts that exceeded 4x the Mean Absolute 209 Deviation of the tree's branch lengths. This simple threshold proved effective at 210 removing spurious BLAST hits in an unbiased manner. Subsequently, the previously 211 identified false-positives were pruned from our phytab-formatted hit-list (part of PIA's 212 output) with the prune_phytab_using_list.py script, also adapted from Osiris (Oakley et 
213 al., 2014). The resulting list of putative opsins was then converted to FASTA format and 214 sequence redundancy was reduced by removing identical protein sequences with 215 UCLUST (Edgar, 2010). The multiple sequence aligner MAFFT (Yamada et al., 2016) 216 was then invoked to align our filtered putative opsins to a large opsin dataset $(n=910)$ 217 compiled by the Porter Lab (University of Hawaii at Manoa), which includes 218 representatives of the main opsin subfamilies. MAFFT alignment parameters were 219 chosen to prioritize accuracy over speed and to allow for large unalignable regions that 220 can be pervasive with divergent GPCRs ("--ep 0 --genafpair --maxiterate 1000"). 221 Following the alignment procedure, a final phylogenetic reconstruction was undertaken 222 with IQ-tree (Nguyen et al., 2015) for characterization and annotation of our PIA223 identified putative opsins. IQ-tree compares favorably to alternatives (e.g. RAXML, 224 FastTree, etc.) in recent benchmarks (Zhou et al., 2017), while also providing a more 225 extensive choice of evolutionary models for phylogenetic inference. After proper 226 consideration, IQ-tree was selected given that evolutionary model choice is important 227 and its choice would be limited in alternative software. Choosing an appropriate model 228 is especially relevant when inferring phylogenetic relationships in protein families with 229 both highly conserved domains and hypervariable regions (e.g. opsins). The IQ-tree 230 analysis was run with a LG general amino acid replacement matrix under a FreeRate 231 model with 10 rate categories and empirical base frequencies (LG+R10+F; Le \& 232 Gascuel, 2008; Soubrier et al., 2012) as suggested by ModelFinder (Kalyaanamoorthy 233 et al., 2017). Branch support was assessed in tripartite by Ultra-fast bootstrap 234 approximation (UFBoot; 10,000 replicates), a Shimodaira-Hasegawa-like approximate 235 likelihood ratio test (SH-aLRT; 10,000 replicates), and an approximate Bayes test 236 (Guindon et al., 2010; Anisimova et al., 2011; Minh et al., 2013).

Finally, the tool HHBlits 'HMM-HMM-based lightning-fast iterative sequence search' (Remmert et al., 2012) was used to confirm opsin identity based on profile 240 HMMs using Uniclust30 (Mirdita et al., 2017) as the reference database. HHBlits was 241 chosen as it incorporates highly sensitive sequence search methods (HMMs) in a fast 242 and more accurate manner compared to other sequence search tools like PSI-BLAST 243 (Remmert et al., 2012). 
245 Genomic Architecture of Annotated Opsins

Proteins encoded in the transcriptomes analyzed may not have corresponding annotations in public databases. Therefore, to validate our pipeline, the exon-intron architecture of the opsin genes obtained from transcriptomic data (see above) was annotated de novo using the recently assembled genomes of $H$. azteca (GCA_000764305.2; accession date: 20-07-2017) and D. pulex (GCA_000187875.1; accessed on 20-07-2017). The Benchmarking set of Universal Single-Copy Orthologs (BUSCO version 3; Simão et al., 2015) was used to ensure an adequate completeness of the genomes used for transcriptome/genome comparison. BUSCO provides quantitative measures for the assessment of genome assembly based on evolutionarilyinformed expectations of gene content from near-universal single-copy orthologs selected from OrthoDB v9. The tblastn algorithm v2.2.29+ was then used with default parameters in order to discriminate between exonic and intronic regions along the genomic scaffolds. When a significant blast hit was found (similarity > 80\%; e-value < $\left.10^{-8}\right)$, the corresponding genomic region was annotated as exonic, or protein coding/expressed region. DNA regions located between two consecutive exons in the same genomic scaffold (chromosome) but with no corresponding counterpart in the expressed RNA were considered as introns. In addition, the nucleotide coding sequence of each putative opsin were mapped to their respective genomes using the spliced aligner HISAT2 (Kim et al., 2015). The mapping was done without penalties for non-canonical splicing using the following command and arguments: "hisat2 $-f-x$ index input.cds.fasta --score-min L,0,-4 --pen-noncansplice 3 -S output.sam". Plots of the gene architecture and the exon length distribution were subsequently completed using the Integrated Genome Browser (Freese et al., 2016) and the software package Mathematica v.11.1 (Wolfram Inc., USA).

271

\section{Results}


Hyalella azteca's transcriptome assembly recovered 243,398 contigs with a 275 mean sequence length of 1033.04 base pairs (Table 2). Of these, 61,401 sequences 276 contained Open Reading Frames (ORFs) designating them as putative protein-coding 277 genes. Similarly, our de novo transcriptome for D. pulex was comprised of 187,310 278 contigs with a mean sequence length of 848.76. Despite the higher number of 279 assembled contigs and the lower mean sequence length, D. pulex's transcriptome 280 contained 38,157 sequences with ORFs. Additional metrics for our de novo 281 transcriptomes, as well as for the reference genomes, are given in Table 2.

283 Table 2. Summary statistics for the de novo transcriptome assemblies produced as part of this study and 284 the corresponding genome assemblies.

\begin{tabular}{|c|c|c|c|c|}
\hline \multirow[b]{2}{*}{ Metric } & \multicolumn{2}{|c|}{ Hyalella azteca } & \multicolumn{2}{|c|}{ Daphnia pulex } \\
\hline & Genome & Transcriptome & Genome & Transcriptome \\
\hline $\begin{array}{c}\text { Number of } \\
\text { sequences/contigs }\end{array}$ & 23426 & 243398 & 18989 & 187310 \\
\hline $\begin{array}{l}\text { Longest sequence/contig } \\
\qquad \text { (bp) }\end{array}$ & 2207822 & 16780 & 528830 & 27096 \\
\hline Number of bases & 550886000 & 251440760 & 197206000 & 158981525 \\
\hline $\begin{array}{c}\text { Mean transcript/contig } \\
\text { length (bp) }\end{array}$ & 23404 & 1033.04 & 8352 & 848.76 \\
\hline $\begin{array}{c}\text { Number of } \\
\text { transcripts/contigs }>1,000 \\
\text { bp long }\end{array}$ & 14563 & 73869 & 16743 & 42717 \\
\hline $\begin{array}{c}\text { Number of } \\
\text { transcripts/contigs }>10,000 \\
\text { bp long }\end{array}$ & 7614 & 157 & 2854 & 179 \\
\hline $\begin{array}{c}\text { Number of transcripts with } \\
\text { ORFs }\end{array}$ & & 61401 & & 38157 \\
\hline Mean ORF percent & & 45.73 & & 50.22 \\
\hline N50 & 114415 & 1929 & 49250 & 1404 \\
\hline N30 & & 3213 & & 2588 \\
\hline N10 & & 5560 & & 5122 \\
\hline GC content & 0.38 & 0.42 & 0.40 & 0.39 \\
\hline
\end{tabular}


we were able to find 990 (92.48\%) complete sequences of the 1066 arthropod genes used for benchmarking. An additional 49 (4.6\%) were also present as fragmented sequences, and only 27 (2.6\%) were not found. Similarly, D. pulex's transcriptome was 291 found to be nearly complete with 1048 (98.4\%) full-length BUSCO genes, 16 (1.5\%) 292 fragmented, and a marginal 2 (0.1\%) missing. The reference genomes selected for validation were rather complete as well, with over $90 \%$ of the BUSCO genes being 294 found complete. Interestingly, the proportion of missing BUSCOs was slightly higher for 295 the genomic data compared to the transcriptomic data (Table 3).

Table 3. Results of transcriptome completeness assessment by Benchmarking Universal Single-Copy Orthologs (BUSCO) using OrthoDB's Arthropoda database of orthologous genes.

\begin{tabular}{c|c|c|c|c|c} 
Species & Dataset & $\begin{array}{c}\text { Complete } \\
\text { BUSCOs }\end{array}$ & $\begin{array}{c}\text { Fragmented } \\
\text { BUSCOs }\end{array}$ & Missing BUSCOs & $\begin{array}{c}\text { Total BUSCOs } \\
\text { Searched }\end{array}$ \\
\hline \multirow{2}{*}{ Hyalella azteca } & Genome & $970(91.0 \%)$ & $29(2.7 \%)$ & $67(6.3 \%)$ & \\
& Transcriptome & $990(92.8 \%)$ & $49(4.6 \%)$ & $27(2.6 \%)$ & 1066 \\
\hline \multirow{2}{*}{ Daphnia pulex } & Genome & $1038(97.3 \%)$ & $9(0.8 \%)$ & $19(1.9 \%)$ & \\
& Transcriptome & $1048(98.4 \%)$ & $16(1.5 \%)$ & $2 \quad(0.1 \%)$ & \\
\hline
\end{tabular}
single FASTA file of amino acid sequences per transcriptome. This file contains the 302 transcripts that remain after the removal of spurious BLAST hits and the merging/removal of duplicated and fragmented sequences, and should only contain 304 those that are closely related to the gene of interest (i.e. opsins). This output can then 305 be piped to a final step for functional annotation by phylogenetic inference. In our case, putative opsin sequences for both species were aligned to a curated dataset of different opsin types. This final step resulted in a large phylogeny (Fig. 1) where opsins are classified based on their phylogenetic position.

311 in Hyalella azteca and Daphnia pulex.

Species
Long-wavelength sensitive
Peropsins I Neuropsins I
Opsin-like transcripts 


\begin{tabular}{|c|c|c|c|c|c|c|c|c|c|c|}
\hline & \multirow[b]{2}{*}{ Genes } & \multirow[b]{2}{*}{ Isoforms } & \multirow[b]{2}{*}{ Genes } & \multirow[b]{2}{*}{ Isoforms } & \multicolumn{2}{|c|}{ Encephalopsins } & \multirow[b]{2}{*}{ Genes } & \multirow[b]{2}{*}{ Isoforms } & \multicolumn{2}{|c|}{ (Outgroups) } \\
\hline & & & & & Genes & Isoforms & & & Genes & Isoforms \\
\hline $\begin{array}{c}\text { Hyalella } \\
\text { azteca }\end{array}$ & 1 & 2 & 3 & 3 & 0 & 0 & 0 & 0 & 1 & 1 \\
\hline $\begin{array}{c}\text { Daphnia } \\
\text { pulex }\end{array}$ & 5 & 15 & 3 & 24 & 0 & 0 & 2 & 4 & 1 & 1 \\
\hline
\end{tabular}

Following Trinity's definition of assembled genes/isoforms, Hyalella azteca's 314 transcriptome contained 1 SWS/UV opsin (2 isoforms), 3 LWS opsins (3 isoforms), and 3151 opsin-like GPCRs (Fig. 2; Table 4) that were identified by our pipeline. On the other 316 hand, Daphnia pulex transcriptome 2 different SWS opsins (4 isoforms), 6 LWS opsins 317 (35 isoforms), 2 melanopsins (4 isoforms), and 1 opsin-like transcript that was placed 318 within the outgroup clade (Fig. 2; Table 4).

Identity results of the HHBlits search using iterative pairwise alignments and 321 profile HHMs are summarized in Table 5, along with the inferred classification of each 322 putative opsin transcript based on their respective placement in the phylogeny (Figs. 323 12). Additionally, each sequence entry was annotated as visual or non-visual based on 324 the sequence homology inferred by both methods (represented by a black box when 325 both methods are in agreement; and a gray box when HMMs fail to identify them as a 326 visual opsin; Table 5). 
Table 5. Identification and classification of Hyalella azteca and Daphnia pulex opsins based on phylogenetic inference and HMM profile

328 alignments. Putative visual opsins are marked in black, under the "Visual" column, when both methodologies are in agreement and in gray when they differ.

\begin{tabular}{|c|c|c|c|c|c|c|c|c|}
\hline Species & Sequence ID & $\begin{array}{l}\text { Phylogeny } \\
\text { Clade }\end{array}$ & Visual & $\begin{array}{l}\text { HHBli } \\
\text { Prob. }\end{array}$ & E-value & P-value & Score & Hit ID \\
\hline \multirow[t]{6}{*}{ Hyalella azteca } & Haz_DN255_c1_g1_i1 & OUTGROUP & & 100.0 & $1.2 \mathrm{E}-66$ & 4.3E-72 & 483.4 & A0A0P5Y4K7_9CRUS Putative Tachykinin peptides receptor 99D (Fragment) OS=Daphnia \\
\hline & Haz_DN34101_c1_g4_i2 & SWS & & 100.0 & $1.2 \mathrm{E}-77$ & 4.3E-83 & 540.3 & OPSL_LIMPO Lateral eye opsin OS=Limulus polyphemus PE=1 SV=1 \\
\hline & Haz_DN34101_c1_g6_i4 & SWS & & 100.0 & $1.9 \mathrm{E}-76$ & $6.9 \mathrm{E}-82$ & 541.7 & OPSL_LIMPO Lateral eye opsin OS=Limulus polyphemus PE=1 SV=1 \\
\hline & Haz_DN31505_c2_g2_i1 & LWS & & 100.0 & $8.8 \mathrm{E}-80$ & $3.1 \mathrm{E}-85$ & 550.9 & OPSL_LIMPO Lateral eye opsin OS=Limulus polyphemus $\mathrm{PE}=1 \mathrm{SV}=1$ \\
\hline & Haz_DN31505_c2_g4_i1 & LWS & & 100.0 & $3.8 \mathrm{E}-80$ & $1.4 \mathrm{E}-85$ & 555.0 & OPSL_LIMPO Lateral eye opsin OS=Limulus polyphemus PE=1 SV=1 \\
\hline & Haz_DN44548_c0_g1_i1 & LWS & & 100.0 & $6.9 \mathrm{E}-72$ & 2.5E-77 & 497.4 & OPSL_LIMPO Lateral eye opsin OS=Limulus polyphemus $\mathrm{PE}=1 \mathrm{SV}=1$ \\
\hline \multirow[t]{23}{*}{ Daphnia pulex } & Dpu_DN105553_c0_g1_i1 & MELANOPSIN & & 100.0 & $8 \mathrm{E}-52$ & $2.8 \mathrm{E}-57$ & 336.2 & H0UT82_CAVPO Uncharacterized protein OS=Cavia porcellus GN=GALR1 PE=3 SV=1 \\
\hline & Dpu_DN16067_c1_g1_i1 & LWS & & 100.0 & $6.3 \mathrm{E}-74$ & 2.2E-79 & 524.1 & A0A0P5USN7_9CRUS Class a rhodopsin g-protein coupled receptor gprop1 OS=Daphnia m \\
\hline & Dpu_DN16472_c1_g1_i1 & OUTGROUP & & 100.0 & $1.8 \mathrm{E}-73$ & $6.2 \mathrm{E}-79$ & 520.2 & A0A0P5Y4K7_9CRUS Putative Tachykinin peptides receptor 99D (Fragment) OS=Daphnia \\
\hline & Dpu_DN21293_c0_g1_i1 & MELANOPSIN & & 100.0 & 3.4E-40 & $1.3 \mathrm{E}-45$ & 291.9 & A0A0P5EI05_9CRUS Class a rhodopsin g-protein coupled receptor gprop2 OS=Daphnia mag \\
\hline & Dpu_DN21293_c0_g2_i1 & MELANOPSIN & & 100.0 & 3.6E-62 & 1.3E-67 & 446.6 & A0A1A6GZZ2_NEOLE Uncharacterized protein OS=Neotoma lepida GN=A6R68_00284 PE \\
\hline & Dpu_DN21293_c0_g2_i2 & MELANOPSIN & & 100.0 & $3.9 \mathrm{E}-38$ & $1.4 \mathrm{E}-43$ & 282.8 & A0A0F8BMY2_LARCR Melanopsin-B OS=Larimichthys crocea GN=EH28_08950 PE=3 S \\
\hline & Dpu_DN22657_c1_g4_i1 & SWS/UV & & 100.0 & $1.7 \mathrm{E}-79$ & $6.1 \mathrm{E}-85$ & 557.5 & OPSL_LIMPO Lateral eye opsin OS=Limulus polyphemus PE=1 SV=1 \\
\hline & Dpu_DN23719_c0_g2_i1 & SWS/UV & & 100.0 & $1.9 \mathrm{E}-78$ & $6.8 \mathrm{E}-84$ & 549.4 & A0A0P5RD30_9CRUS Class a rhodopsin g-protein coupled receptor gprop2 OS=Daphnia me \\
\hline & Dpu_DN23719_c0_g2_i2 & SWS/UV & & 100.0 & 4.5E-78 & $1.6 \mathrm{E}-83$ & 544.5 & A0A0P5RD30_9CRUS Class a rhodopsin g-protein coupled receptor gprop2 OS=Daphnia me \\
\hline & Dpu_DN23719_c0_g3_i1 & SWS/UV & & 100.0 & $2 \mathrm{E}-78$ & 7.4E-84 & 548.4 & A0A0P5RD30_9CRUS Class a rhodopsin g-protein coupled receptor gprop2 OS=Daphnia me \\
\hline & Dpu_DN25251_c0_g2_i1 & SWS/UV & & 100.0 & 2.6E-77 & 9.3E-83 & 532.3 & OPSL_LIMPO Lateral eye opsin OS=Limulus polyphemus PE=1 SV=1 \\
\hline & Dpu_DN25251_c0_g2_i3 & SWS/UV & & 100.0 & 3.4E-78 & $1.2 \mathrm{E}-83$ & 547.0 & OPSL_LIMPO Lateral eye opsin OS=Limulus polyphemus PE=1 SV=1 \\
\hline & Dpu_DN25251_c0_g2_i4 & SWS/UV & & 100.0 & $1.9 \mathrm{E}-77$ & $6.8 \mathrm{E}-83$ & 533.3 & OPSL_LIMPO Lateral eye opsin OS=Limulus polyphemus PE=1 SV=1 \\
\hline & Dpu_DN25251_c0_g2_i5 & SWS/UV & & 100.0 & $8.2 \mathrm{E}-77$ & 2.9E-82 & 529.8 & OPSL_LIMPO Lateral eye opsin OS=Limulus polyphemus PE=1 SV=1 \\
\hline & Dpu_DN25251_c0_g3_i1 & SWS/UV & & 100.0 & 2.6E-77 & $9.1 \mathrm{E}-83$ & 537.5 & OPSL_LIMPO Lateral eye opsin OS=Limulus polyphemus PE=1 SV=1 \\
\hline & Dpu_DN25293_c2_g1_i1 & SWS/UV & & 100.0 & $5.7 \mathrm{E}-48$ & $2.2 \mathrm{E}-53$ & 330.3 & OPSL_LIMPO Lateral eye opsin OS=Limulus polyphemus PE=1 SV=1 \\
\hline & Dpu_DN25293_c2_g1_i3 & SWS/UV & & 100.0 & $2 \mathrm{E}-78$ & $7.1 \mathrm{E}-84$ & 548.9 & OPSL_LIMPO Lateral eye opsin OS=Limulus polyphemus PE=1 SV=1 \\
\hline & Dpu_DN25293_c3_g1_i1 & SWS/UV & & 100.0 & 5.7E-35 & $2 \mathrm{E}-40$ & 227.3 & D0E2W5_CHICK Uncharacterized protein OS=Gallus gallus GN=OPNVA PE=2 SV=1 \\
\hline & Dpu_DN25293_c4_g1_i1 & SWS/UV & & 100.0 & $1.1 \mathrm{E}-75$ & 3.9E-81 & 538.2 & OPSL_LIMPO Lateral eye opsin OS=Limulus polyphemus PE=1 SV=1 \\
\hline & Dpu_DN25293_c7_g1_i1 & SWS/UV & & 100.0 & $1.4 \mathrm{E}-33$ & $5.1 \mathrm{E}-39$ & 219.0 & OPSL_LIMPO Lateral eye opsin OS=Limulus polyphemus PE=1 SV=1 \\
\hline & Dpu_DN25377_c0_g1_i1 & LWS & & 100.0 & $6.3 \mathrm{E}-75$ & 2.2E-80 & 517.7 & A0A0P5USN7_9CRUS Class a rhodopsin g-protein coupled receptor gprop1 OS=Daphnia m \\
\hline & Dpu_DN25377_c0_g1_i10 & LWS & & 100.0 & $3.1 \mathrm{E}-62$ & $1.1 \mathrm{E}-67$ & 425.5 & A0A0P5KGY5_9CRUS Class a rhodopsin g-protein coupled receptor gprop1 OS=Daphnia m \\
\hline & Dpu_DN25377_c0_g1_i11 & LWS & & 100.0 & $4.1 \mathrm{E}-76$ & 1.4E-81 & 528.5 & A0A0P5USN7_9CRUS Class a rhodopsin g-protein coupled receptor gprop1 OS=Daphnia m \\
\hline
\end{tabular}


Dpu_DN25377_c0_g1_i12 LWS

Dpu_DN25377_c0_g1_i4 LWS

Dpu_DN25377_c0_g1_i5 LWS

Dpu_DN25377_c0_g1_i6 LWS

Dpu_DN25377_c0_g1_i7 LWS

Dpu_DN25377_c0_g1_i8 LWS

Dpu_DN25377_c0_g1_i9 LWS

Dpu_DN25377_c0_g2_i1 LWS

Dpu_DN25377_c0_g2_i11 LWS

Dpu_DN25377_c0_g2_i2 LWS

Dpu_DN25377_c0_g2_i3 LWS

Dpu_DN25377_c0_g2_i5 LWS

Dpu_DN25377_c0_g2_i6 LWS

Dpu_DN25377_c0 g2 i8 LWS

Dpu_DN25377_c0_g2_i9 LWS

Dpu_DN25506_c1_g1_i1 LWS

Dpu_DN25506_c1_g1_i2 LWS

Dpu_DN25506_c3_g1_i1 LWS

Dpu_DN25506_c3_g1_i3 LWS

Dpu_DN25506_c4_g1_i1 LWS

Dpu_DN27815_c0_g1_i1 SWS/UV

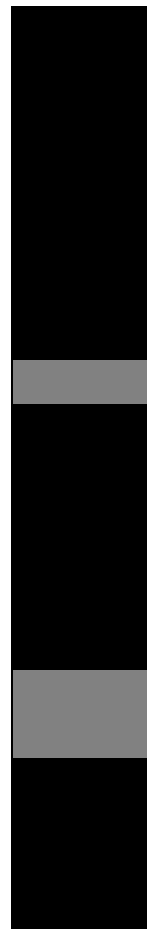

\begin{tabular}{l|l|l|l|}
100.0 & $1.4 \mathrm{E}-75$ & $4.9 \mathrm{E}-81$ & 523.3
\end{tabular}

\begin{tabular}{l|l|l}
100.0 & $1 \mathrm{E}-56$ & $3.6 \mathrm{E}-62$
\end{tabular}

\begin{tabular}{l|l|l}
100.0 & $5.3 \mathrm{E}-82$ & $1.8 \mathrm{E}-87$
\end{tabular}

\begin{tabular}{l|l|l}
100.0 & $8 \mathrm{E}-77$ & $2.8 \mathrm{E}-82$
\end{tabular}

\begin{tabular}{l|l|l}
100.0 & $2 \mathrm{E}-76$ & $6.8 \mathrm{E}-82$
\end{tabular}

$100.0 \quad 1.1 \mathrm{E}-80 \quad 3.7 \mathrm{E}-86$

\begin{tabular}{l|l|l}
100.0 & $4 \mathrm{E}-77$ & $1.4 \mathrm{E}-82$
\end{tabular}

\begin{tabular}{l|l|l}
100.0 & $4.5 \mathrm{E}-76$ & $1.6 \mathrm{E}-81$
\end{tabular}

\begin{tabular}{l|l|l}
99.9 & $8.6 \mathrm{E}-29$ & $3.3 \mathrm{E}-34$
\end{tabular}

\begin{tabular}{l|l|l}
100.0 & $1.7 \mathrm{E}-75$ & $6 \mathrm{E}-81$
\end{tabular}

\begin{tabular}{l|l|l}
100.0 & $1.2 \mathrm{E}-75$ & $4.2 \mathrm{E}-81$
\end{tabular}

\begin{tabular}{l|l|l}
100.0 & $4.4 \mathrm{E}-76$ & $1.5 \mathrm{E}-81$
\end{tabular}

\begin{tabular}{l|l|l|}
100.0 & $4.2 \mathrm{E}-77$ & $1.4 \mathrm{E}-82$
\end{tabular}

\begin{tabular}{l|l|l}
100.0 & $9.3 \mathrm{E}-76$ & $3.2 \mathrm{E}-81$
\end{tabular}

$100.0 \quad 4.3 \mathrm{E}-76 \quad 1.5 \mathrm{E}-81$

\begin{tabular}{l|l|l}
100.0 & $8.9 \mathrm{E}-35$ & $3.2 \mathrm{E}-40$
\end{tabular}

$100.0 \quad 1.1 \mathrm{E}-34 \quad 3.9 \mathrm{E}-40$

\begin{tabular}{l|l|l}
100.0 & $4.3 \mathrm{E}-80$ & $1.4 \mathrm{E}-85$
\end{tabular}

$100.0 \quad 1.1 \mathrm{E}-60 \quad 4 \mathrm{E}-66$

$100.0 \quad 2.3 \mathrm{E}-48 \quad 8.2 \mathrm{E}-54$

\begin{tabular}{l|l|l|}
100.0 & $9.5 \mathrm{E}-71$ & $3.4 \mathrm{E}-76$
\end{tabular}
386.9

558.9

534.7

529.2

552.3

533.7

528.0

200.2

524.9

525.7

529.3

535.1

523.9

528.5

222.0

221.4

521.3

401.9

312.8

496.1
A0A0P5USN7_9CRUS Class a rhodopsin g-protein coupled receptor gprop1 OS=Daphnia m A0A0P5KGY5_9CRUS Class a rhodopsin g-protein coupled receptor gprop1 OS=Daphnia m A0A0P5USN7_9CRUS Class a rhodopsin g-protein coupled receptor gprop1 OS=Daphnia m A0A0P5USN7_9CRUS Class a rhodopsin g-protein coupled receptor gprop1 OS=Daphnia m A0A0P5USN7_9CRUS Class a rhodopsin g-protein coupled receptor gprop1 OS=Daphnia m A0A0P5USN7_9CRUS Class a rhodopsin g-protein coupled receptor gprop1 OS=Daphnia m A0A0P5USN7_9CRUS Class a rhodopsin g-protein coupled receptor gprop1 OS=Daphnia m A0A0P5USN7_9CRUS Class a rhodopsin g-protein coupled receptor gprop1 OS=Daphnia m E9FX22_DAPPU Octopamine receptor beta-2-like protein OS=Daphnia pulex GN=DAPPUD A0A0P5USN7_9CRUS Class a rhodopsin g-protein coupled receptor gprop1 OS=Daphnia m A0A0P5USN7_9CRUS Class a rhodopsin g-protein coupled receptor gprop1 OS=Daphnia m A0A0P5USN7_9CRUS Class a rhodopsin g-protein coupled receptor gprop1 OS=Daphnia m A0A0P5USN7_9CRUS Class a rhodopsin g-protein coupled receptor gprop1 OS=Daphnia m A0A0P5USN7_9CRUS Class a rhodopsin g-protein coupled receptor gprop1 OS=Daphnia m A0A0P5USN7_9CRUS Class a rhodopsin g-protein coupled receptor gprop1 OS=Daphnia m E6ZHB1_DICLA Beta-1 adrenergic receptor OS=Dicentrarchus labrax GN=ADRB1 PE=3 S E6ZHB1_DICLA Beta-1 adrenergic receptor OS=Dicentrarchus labrax GN=ADRB1 PE=3 S A0A0P4XHY0_9CRUS Class a rhodopsin g-protein coupled receptor gprop1 OS=Daphnia m A0A0P5KGY5_9CRUS Class a rhodopsin g-protein coupled receptor gprop1 OS=Daphnia m OPSL_LIMPO Lateral eye opsin OS=Limulus polyphemus $\mathrm{PE}=1 \mathrm{SV}=1$

A0A0P5RD30_9CRUS Class a rhodopsin g-protein coupled receptor gprop2 OS=Daphnia m 
Every protein sequence predicted using the modified PIA pipeline gave at least one significant TBLASTN hit both in the $D$. pulex and $H$. azteca reference genomes. The observed distribution of introns within opsin genes appears to be variable both within and between genomes. To illustrate this variation, the Intron-Exon gene structure patterns of representative opsins were further characterized. The genomic region encoding for the SWS/UV opsin gene spanned about $4 \mathrm{~kb}$ in Hyalella and presented an extremely partitioned structure formed by at least 7 different exons (Figure 3). Interestingly, some LWS opsins within the $H$. azteca genome were located on the antisense strand and appear to be duplicated retrogenes (Figure 3; see Discussion). Both SWS/UV and LWS opsins were also arranged following disparate gene architectures in the D. pulex genome (Figure 4). LWS opsins presented slightly shorter introns on average than SWS/UV opsins, but the presence of gene duplications and genes with numerous introns were identified in most cases. Exon size distribution had a similar shape in $D$. pulex and $H$. azteca, being multimodal for both genomes (Figure 5). Nevertheless, $H$. azteca had a larger average exon size (Mean $=410 \mathrm{bp}$; Median $=235$ bp) than D. pulex (Mean $=225 \mathrm{bp}$; Median = 164). Mapping results in SAM format are available for download from the following figshare repository http://figshare.com/xxxxxx.

\section{Discussion}

Our results demonstrate the power of incorporating phylogenetic annotation towards the characterization and interpretation of large transcriptomic datasets of nonmodel organisms. Annotations via simple sequence similarity based methods like BLAST alone can result in false positives including, but not limited to, functional diversification following gene duplication events, domain shuffling, or even existing database errors (review Sjölander, 2004). Using the modified version of PIA has allowed for the rapid and automated identification of false positives among the putative visual opsins curated for the two species of crustaceans, Hyalella azteca and Daphnia pulex. The modified Phylogenetically Informed Annotation pipeline was able to 
363

364

365

366

367

368

369

370

371

372

373

374

375

376

377

378

379

380

381

382

383

384

385

386

387

388

389

390

391

392

393

successfully identify and filter opsins from the de novo transcriptomes in a fully automated manner with minimal manual curation. This automation is made possible mainly by the modifications and wrapper scripts that converted PIA from a Galaxy workflow to a command-line one, which effectively increases the scalability of the pipeline allowing for the identification of opsins (and other genes) from multiple transcriptomic datasets through simple scripting. Theoretically, this would allow for the annotation of dozens if not hundreds of transcriptomes at a time without the need of the excessive time-costs that a graphical user interface and manual curation of hundreds of phylogenetic trees would imply. Further improvements are certainly possible, particularly in terms of parallelization for its use in High Performance Computing environments for even greater computing speeds. Nevertheless, the current pipeline is dependent on its individual components and would thus require those to be made compatible with parallelization.

The initial hits recovered by a BLAST search using the original PIA opsin dataset recovered 11 putative opsin isoforms for $H$. azteca and 51 for $D$. pulex. Our pipeline removed $54.5 \%$ and $23.5 \%$ of those as spurious hits (Table 4 ) based on the chosen branch length thresholds. These thresholds can easily be adjusted for increased/decreased conservativeness if deemed necessary, which should be assessed on a gene-to-gene basis. The final phylogenetic inference took a step further by classifying these opsins in statistically supported functional clades (Figs. 1-2), which allowed for the determination of their putative photoreceptive roles. Both $\mathrm{H}$. azteca and D. pulex transcriptomes were generated from whole organism RNA extractions. As opsins are known to function in various cells and tissues of arthropods, as well as the retina (e.g. Lampel et al., 2005; Tong et al., 2009), it is likely that the opsin groups identified here are expressed across several tissue types. Non-visual opsins can be readily identified via phylogenetic inference provided that appropriate reference sequences are included in the multiple sequence alignments (for example, Fig. 3). HMM alignments were also used to confirm protein identities as well as to compare with the results of the phylogenetic annotation. HHMs were able to pair most putative visual opsins to the lateral compound eye opsins of arthropods for both species and, in the 
394 case of $D$. pulex, specifically to Daphnia class A rhodopsins. While there were a few 395 discrepancies among annotation methods with regard to visual opsins (r-opsins) and melanopsins, this could be explained by their common origin (Porter et al., 2012). Melanopsins are very similar to the r-opsins found in invertebrates (Provencio et al., 1998, 2000) and can couple to similar signaling cascades (Isoldi et al., 2005; Panda et al., 2005; Qiu et al., 2005), likely contributing to the observed contradictory results depending on the methods. In fact, the similarities between these opsin types are evident in our phylogenetic trees (Figs. 1 \& 2), showing a well-supported clade of arthropod UV opsins nested within the melanopsin clade. Partial sequences or existing database errors could also be a contributing factor to which BLAST and HMM approaches are more sensitive. Even though the HMM searches were not able to determine the functional classification of the opsins in terms of spectral sensitivity, they were confirmed as visual opsins (Table 5). Our results further support the notion that integrated annotation methods are advantageous and recommended to confirm the robustness of findings and annotations.

Opsin repertoire and spectral sensitivities

There are several subgroups of rhabdomeric visual opsins responsible for vision in crustaceans, each with characteristic absorption spectra when bound to a 414 chromophore (Kashiyama et al., 2009; Henze \& Oakley, 2015). The number and type of opsins found throughout Crustacea can range greatly, partially owing to differences in methodologies- with no present homologs found in freshwater Bathynellacea (Kim et al., 417 2017), one or two SWS visual opsins found in species of deep-sea shrimp (Wong et al., 418 2015), and brachyuran crabs (Sakamoto et al., 1996), and as many as 33 identified in 419 stomatopods (Porter et al., 2009, 2013). The number of opsins and corresponding 420 spectral sensitivity of an organism appears to correlate with its life-history, habitat, and 421 the ecological niche it may occupy (Marshall et al., 2015; Stieb et al., 2017). This study 422 represents the first transcriptomic exploration of $H$. azteca's opsin repertoire, which 423 revealed several putative visual opsins (Fig. 2; Table 4). Hyalella azteca is a freshwater epibenthic amphipod commonly used as a bioindicator species. Though further 
425 evidence is required to make inferences regarding the expression and functionality of 426 these putative opsins, the ability to differentiate between short and long wavelengths 427 would allow $H$. azteca to discern between direct and reflected light from the benthos. 428 Direct sunlight (or moonlight) tends to be abundant in short-wavelengths (<450nm) 429 whereas reflected light from sources like leaves and sediment tends to be shifted 430 towards longer (>450nm) wavelengths (Menzel, 1979). Our analyses revealed four 431 distinct opsin genes (one SWS/UV and three LWS) expressed in its transcriptome, 432 suggest that $H$. azteca may be capable of discriminating between the aforementioned 433 light sources. The authors hypothesize that if $\mathrm{H}$. azteca does possess functional SWS 434 and LWS visual opsins, this distinction could serve as an important environmental cue 435 influencing their response to a variety of abiotic and biotic factors (e.g. refugia, 436 vegetation, predators, prey). However, the authors note additional studies incorporating 437 electroretinographic analyses are needed to confirm whether $H$. azteca can indeed 438 discriminate between different wavelengths of light as the transcriptomic data suggests. 439 Fewer opsins were found to be expressed in $H$. azteca compared to $D$. pulex, which is 440 not surprising given the expansive opsin repertoire previously described for Daphnia 441 (Colbourne et al., 2011; Brandon et al., 2017). Daphnia has both a simple and 442 compound eyes, which may contribute to the relatively large number of opsin isoforms 443 expressed. Differences have been found in the number and type of opsin genes 444 expressed among eye forms within the ostracod Skogsberia lerneri (Oakley \& Huber, 445 2004) and similarly hypothesized for Daphnia (Brandon et al., 2017).

The subset of identified rhabdomeric opsins expressed in the D. pulex 448 transcriptome allows for comparisons to prior studies characterizing the range of opsin 449 types found in the D. pulex genome (Colbourne et al., 2011; Brandon et al., 2015). 450 Colbourne et al. (2011) reported 25 medium- (MWS) and long-wavelength sensitive 451 (LWS) opsin genes as present in the D. pulex genome, but only 3 LWS opsin genes 452 (and 24 isoforms) were identified in our analyses. While it is possible that the additional 453 opsin classes identified in previous genomic investigations were not expressed in the 454 current $D$. pulex dataset, it is also possible these discrepancies are due to differences in 455 classification schemes across Arthropoda, with 'blue-green' wavelengths currently 
456 grouped under SWS. An alternative explanation is that separate genes are being 457 considered isoforms of each other by Trinity during assembly process. Considering the 458 large number of "isoforms" and low number of "genes" identified in the D. pulex 459 transcriptome, in contrast with previous genomic investigations (i.e.Brandon et al., 460 2017), this is likely a contributing factor to this observed discrepancy.

Genomic architecture and opsin gene duplications

Gene duplications play a fundamental role in genome evolution (Ohno, 1970; Kondrashov et al., 2002), with replicates occasionally evolving new biological functions (Zhang, 2003; Pegueroles et al., 2013). Some of these genome duplications are considered as pseudogenes, loci whose nucleotide sequences are similar to a normal gene but that do not produce a functional product when translated. The "unprocessed" pseudogenes, have all the normal parts of a protein-coding gene, but generally are nonfunctional due to coding errors (Lynch \& Force, 1999). The so-called "processed" pseudogenes or retrogenes lack the non-coding introns present in the original gene,

472 and are thought to arise from mRNA reinserted into the genome by reverse transcription 473 (Betrán \& Long, 2002). Some retrogenes have been found to be actively transcribed 474 and the RNA product can be further processed to give two different molecules of RNA 475 of smaller size that form elaborate secondary structures. These RNA regulatory 476 molecules can control a variety of key genes involved in the regulation of the cell cycle 477 and in cell growth (Tutar, 2012; Wen et al., 2012). Intron-less opsin genes, such as the 478 LWS opsins our analyses identified in H. azteca (Fig. 3), have evolved in various 479 metazoans (including crustaceans) and are thought to be functional photoproteins 480 (Morris et al., 1993; Fitzgibbon et al., 1995; Porter et al., 2007; Liegertová et al., 2015), 481 though it has been postulated that the expression of retrotransposed opsins is a form of 482 transcriptional noise and a byproduct of transcriptional activity in the new genomic 483 region ( $\mathrm{Xu}$ et al., 2016). Opsin diversification and photopigment evolution seems to 484 have been driven by duplicated opsin genes (e.g. Frentiu et al., 2007; Briscoe et al., 485 2010), as is the case of both ocular and extraocular cnidarian photoreceptors 486 (Liegertová et al., 2015). Likewise, a functional LWS retrogene was recently found in the 
arthropod Helicoverpa armigera, though expression was believed to be under temporal compartmentalization and primarily expressed in larval stages (Xu et al., 2016). Our results provide further evidence supporting the importance of retrogenes in the evolution of the opsins gene family.

\section{Concluding remarks}

Our results support the use of integrative phylogenetic annotation rather than just similarity-based approaches. This is an often overlooked but especially important consideration for the study of protein families (e.g. GPCRs) known for having large numbers of isoforms, multiple duplication events, low sequence similarities, and various combinations of highly conserved domains with hypervariable regions. Phylogenetic approaches are not only able to robustly evaluate homology in an evolutionary context, but they can also provide valuable functional information based on recovered branching patterns. In the case of opsins, this functional information can be insightful from a variety perspectives, and aid in the formulation and testing of organismal, ecological, and evolutionary hypotheses. Many of these will be put to the test in the present and forthcoming genomic era, for which efficient and scalable methodologies and pipelines will be paramount.

\section{Acknowledgements}

The authors would like to thank Megan Porter for access to her compilation of reference opsin data, as well as Daniel Speiser and Todd Oakley for allowing us to modify the original PIA tool. JPM was supported by the Philip M. Smith Graduate Research Grant for Cave and Karst Research from the Cave Research Foundation and The Crustacean Society Scholarship in Graduate Studies. This work was partially funded by two grants awarded from the National Science Foundation: Doctoral Dissertation Improvement Grant (\#1701835) awarded to JPM and HBG and Division of Environmental Biology Bioluminescence and Vision grant (DEB-1556059) awarded to HBG. FP acknowledges 
517 project CHALLENGEN (CTM2013-48163) of the Spanish Government and a post518 doctoral contract funded by the Beatriu de Pinos Programme of the Generalitat de 519 Catalunya (2014-BPB-00038). The authors would like to acknowledge the Instructional

520 \& Research Computing Center (IRCC) at Florida International University for providing 521 High Performance Computing resources that have contributed to the research results 522 reported within this article. This is contribution \#xx of the Marine Education and 523 Research Center of the Institute for Water and the Environment at Florida International 524 University.

525

\section{References}

Afgan, E., D. Baker, M. van den Beek, D. Blankenberg, D. Bouvier, M. Čech, J. Chilton, D. Clements, N. Coraor, C. Eberhard, B. Grüning, A. Guerler, J. HillmanJackson, G. Von Kuster, E. Rasche, N. Soranzo, N. Turaga, J. Taylor, A. Nekrutenko, \& J. Goecks, 2016. The Galaxy platform for accessible, reproducible and collaborative biomedical analyses: 2016 update. Nucleic Acids Research 44: W3-W10.

Altschul, S. F., W. Gish, W. Miller, E. W. Myers, \& D. J. Lipman, 1990. Basic local alignment search tool. Journal of Molecular Biology 215: 403-410.

Andrews, S., 2010. FastQC A Quality Control tool for High Throughput Sequence Data. http://www.bioinformatics.babraham.ac.uk/projects/fastqc/

Anisimova, M., M. Gil, J.-F. Dufayard, C. Dessimoz, \& O. Gascuel, 2011. Survey of Branch Support Methods Demonstrates Accuracy, Power, and Robustness of Fast Likelihood-based Approximation Schemes. Systematic Biology 60: 685699.

Arendt, D., K. Tessmar, M.-I. M. de Campos-Baptista, A. Dorresteijn, \& J. Wittbrodt, 2002. Development of pigment-cup eyes in the polychaete Platynereis dumerilii and evolutionary conservation of larval eyes in Bilateria. Development 129: 1143-1154.

Arendt, D., K. Tessmar-Raible, H. Snyman, A. W. Dorresteijn, \& J. Wittbrodt, 2004. Ciliary photoreceptors with a vertebrate-type opsin in an invertebrate brain. Science 306: 869-871.

Betrán, E., \& M. Long, 2002. Expansion of genome coding regions by acquisition of new genes. Genetica 115: 65-80. 
551

Biscontin, A., E. Frigato, G. Sales, G. M. Mazzotta, M. Teschke, C. De Pittà, S. Jarman, B. Meyer, R. Costa, \& C. Bertolucci, 2016. The opsin repertoire of the Antarctic krill Euphausia superba. Marine Genomics 29: 61-68.

Bok, M. J., M. L. Porter, \& D.-E. Nilsson, 2017. Phototransduction in fan worm radiolar eyes. Current Biology 27: R681-R701.

Bolger, A. M., M. Lohse, \& B. Usadel, 2014. Trimmomatic: a flexible trimmer for Illumina sequence data. Bioinformatics 30: 2114-2120.

Brandon, C. S., M. J. Greenwold, \& J. L. Dudycha, 2017. Ancient and Recent Duplications Support Functional Diversity of Daphnia Opsins. Journal of Molecular Evolution 84: 12-28.

Briscoe, A. D., S. M. Bybee, G. D. Bernard, F. Yuan, M. P. Sison-Mangus, R. D. Reed, A. D. Warren, J. Llorente-Bousquets, \& C.-C. Chiao, 2010. Positive selection of a duplicated UV-sensitive visual pigment coincides with wing pigment evolution in Heliconius butterflies. Proceedings of the National Academy of Sciences 107: 3628-3633.

Cock, P. J. A., T. Antao, J. T. Chang, B. A. Chapman, C. J. Cox, A. Dalke, I. Friedberg, T. Hamelryck, F. Kauff, B. Wilczynski, \& M. J. L. de Hoon, 2009. Biopython: freely available Python tools for computational molecular biology and bioinformatics. Bioinformatics 25: 1422-1423.

Colbourne, J. K., M. E. Pfrender, D. Gilbert, W. K. Thomas, A. Tucker, T. H. Oakley, S. Tokishita, A. Aerts, G. J. Arnold, M. K. Basu, D. J. Bauer, C. E. Caceres, L. Carmel, C. Casola, J.-H. Choi, J. C. Detter, Q. Dong, S. Dusheyko, B. D. Eads, T. Frohlich, K. A. Geiler-Samerotte, D. Gerlach, P. Hatcher, S. Jogdeo, J. Krijgsveld, E. V. Kriventseva, D. Kultz, C. Laforsch, E. Lindquist, J. Lopez, J. R. Manak, J. Muller, J. Pangilinan, R. P. Patwardhan, S. Pitluck, E. J. Pritham, A. Rechtsteiner, M. Rho, I. B. Rogozin, O. Sakarya, A. Salamov, S. Schaack, H. Shapiro, Y. Shiga, C. Skalitzky, Z. Smith, A. Souvorov, W. Sung, Z. Tang, D. Tsuchiya, H. Tu, H. Vos, M. Wang, Y. I. Wolf, H. Yamagata, T. Yamada, Y. Ye, J. R. Shaw, J. Andrews, T. J. Crease, H. Tang, S. M. Lucas, H. M. Robertson, P. Bork, E. V. Koonin, E. M. Zdobnov, I. V. Grigoriev, M. Lynch, \& J. L. Boore, 2011. The ecoresponsive genome of Daphnia pulex. Science 331: 555-561.

Crisp, M., \& L. Cook, 2005. Do early branching lineages signify ancestral traits? Trends in Ecology \& Evolution 20: 122-128.

Eddy, S. R., 2011. Accelerated Profile HMM Searches. PLoS computational biology 7: e1002195-e1002195.

Edgar, R. C., 2010. Search and clustering orders of magnitude faster than BLAST. Bioinformatics 26: 2460-2461. 
Engelhardt, B. E., M. I. Jordan, S. T. Repo, \& S. E. Brenner, 2009. Phylogenetic molecular function annotation. Journal of Physics: Conference Series 180: 012024.

Feuda, R., O. Rota-Stabelli, T. H. Oakley, D. Pisani, 2014. The Comb Jelly Opsins and the Origins of Animal Phototransduction. Genome Biology and Evolution 6: 19641971.Feuda, R., F. Marlétaz, M. A. Bentley, P. W.H. Holland, 2016. Conservation, Duplication, and Divergence of Five Opsin Genes in Insect Evolution. Genome Biology and Evolution 8:579-587.

Fitzgibbon, J., A. Hope, S. J. Slobodyanyuk, J. Bellingham, J. K. Bowmaker, \& D. M. Hunt, 1995. The rhodopsin-encoding gene of bony fish lacks introns. Gene 164: 273-277.

Freese, N. H., D. C. Norris, \& A. E. Loraine, 2016. Integrated genome browser: visual analytics platform for genomics. Bioinformatics 32: 2089-2095.

Frentiu, F. D., G. D. Bernard, M. P. Sison-Mangus, A. Van Zandt Brower, \& A. D. Briscoe, 2007. Gene Duplication Is an Evolutionary Mechanism for Expanding Spectral Diversity in the Long-Wavelength Photopigments of Butterflies. Molecular Biology and Evolution 24: 2016-2028.

Fryxel, K. J., \& E. M. Meyerowitz, 1991. The evolution fo rhodopsins and neurotransmitter receptors. Journal of Molecular Evolution 33: 367-378.

Gaudet, P., M. S. Livstone, S. E. Lewis, \& P. D. Thomas, 2011. Phylogenetic-based propagation of functional annotations within the Gene Ontology consortium. Briefings in Bioinformatics 12: 449-462.

Gonzalez, E. R., L. Watling, 2002. Redescription of Hyalella azteca from Its type locality, Vera Cruz, Mexico (Amphipoda:Hyalellidae). Journal of Crustacean Biology 22: 173-183.Grabherr, M. G., B. J. Haas, M. Yassour, J. Z. Levin, D. a Thompson, I. Amit, X. Adiconis, L. Fan, R. Raychowdhury, Q. Zeng, Z. Chen, E. Mauceli, N. Hacohen, A. Gnirke, N. Rhind, F. di Palma, B. W. Birren, C. Nusbaum, K. Lindblad-Toh, N. Friedman, \& A. Regev, 2011. Full-length transcriptome assembly from RNA-Seq data without a reference genome. Nature Biotechnology 29: 644-652.

Gühmann, M., H. Jia, N. Randel, C. Verasztó, L. A. Bezares-Calderón, N. K. Michiels, S. Yokoyama, \& G. Jékely, 2015. Spectral Tuning of Phototaxis by a Go-Opsin in the Rhabdomeric Eyes of Platynereis. Current Biology 25: 2265-2271.

Guindon, S., J.-F. Dufayard, V. Lefort, M. Anisimova, W. Hordijk, \& O. Gascuel, 2010. New Algorithms and Methods to Estimate Maximum-Likelihood Phylogenies: Assessing the Performance of PhyML 3.0. Systematic Biology 59: 307-321.

Haas, B. J., A. Papanicolaou, M. Yassour, M. Grabherr, P. D. Blood, J. Bowden, M. B. Couger, D. Eccles, B. Li, M. Lieber, M. D. Macmanes, M. Ott, J. Orvis, N. Pochet, 
F. Strozzi, N. Weeks, R. Westerman, T. William, C. N. Dewey, R. Henschel, R. D. Leduc, N. Friedman, \& A. Regev, 2013. De novo transcript sequence reconstruction from RNA-seq using the Trinity platform for reference generation and analysis. Nature Protocols 8: 1494-1512.

Henze, M. J., \& T. H. Oakley, 2015. The Dynamic Evolutionary History of Pancrustacean Eyes and Opsins. Integrative and Comparative Biology 55: 830842.

Hering, L., \& G. Mayer, 2014. Analysis of the Opsin Repertoire in the Tardigrade Hypsibius dujardini Provides Insights into the Evolution of Opsin Genes in Panarthropoda. Genome Biology and Evolution 6: 2380-2391.

Imai, H., D. Kojima, T. Oura, S. Tachibanaki, A. Terakita, \& Y. Shichida, 1997. Single amino acid residue as a functional determinant of rod and cone visual pigments. Proceedings of the National Academy of Sciences 94: 2322-2326.

Isoldi, M. C., M. D. Rollag, A. M. de Lauro Castrucci, \& I. Provencio, 2005. Rhabdomeric phototransduction initiated by the vertebrate photopigment melanopsin. Proceedings of the National Academy of Sciences 102: 1217-1221.

Kalyaanamoorthy, S., B. Q. Minh, T. K. F. Wong, A. von Haeseler, \& L. S. Jermiin, 2017. ModelFinder: fast model selection for accurate phylogenetic estimates. Nature Methods 14: 587-589.

Kashiyama, K., T. Seki, H. Numata, \& S. G. Goto, 2009. Molecular Characterization of Visual Pigments in Branchiopoda and the Evolution of Opsins in Arthropoda. Molecular Biology and Evolution 26: 299-311.

Katti, C., K. Kempler, M. L. Porter, A. Legg, R. Gonzalez, E. Garcia-Rivera, D. Dugger, \& B.-A. Battelle, 2010. Opsin co-expression in Limulus photoreceptors: differential regulation by light and a circadian clock. Journal of Experimental Biology 213: 2589-2601.

Kim, D., B. Langmead, \& S. L. Salzberg, 2010. HISAT: a fast spliced aligner with low memory requirements. Nature Methods 12: 357-360.

Kim, B.-M., S. Kang, D.-H. Ahn, J.-H. Kim, I. Ahn, C.-W. Lee, J.-L. Cho, G.-S. Min, \& H. Park, 2017. First insights into the subterranean crustacean Bathynellacea transcriptome: transcriptionally reduced opsin repertoire and evidence of conserved homeostasis regulatory mechanisms. PloS One 12: e0170424.

Kojima, D., A. Terakita, T. Ishikawa, Y. Tsukahara, A. Maeda, \& Y. Shichida, 1997. A novel Go-mediated phototransduction cascade in scallop visual cells. Journal of Biological Chemistry 272: 22979-22982.

Kondrashov, F. A., I. B. Rogozin, Y. I. Wolf, \& E. V. Koonin, 2002. Selection in the evolution of gene duplications. Genome Biology 3: research0008-1. 
663

664

665

666

667

668

669

670

671

672

673

674

675

676

677

678

679

680

681

682

683

684

685

686

687

688

689

690

691

692

693

694

695

696

697

Krogh, A., M. Brown, I. S. Mian, K. Sjolander, \& D. Haussler, 1994. Hidden Markov Models in Computational Biology. Molecular Biology 235: 1501-1531.

Kuwayama, S., H. Imai, T. Hirano, A. Terakita, \& Y. Shichida, 2002. Conserved Proline Residue at Position 189 in Cone Visual Pigments as a Determinant of Molecular Properties Different from Rhodopsins . Biochemistry 41: 15245-15252.

Lampel, J., A. D. Briscoe, \& L. T. Wasserthal, 2005. Expression of UV-, blue-, longwavelength-sensitive opsins and melatonin in extraretinal photoreceptors of the optic lobes of hawkmoths. Cell and Tissue Research 321: 443-458.

Le, S. Q., \& O. Gascuel, 2008. An Improved General Amino Acid Replacement Matrix. Molecular Biology and Evolution 25: 1307-1320.

Liegertová, M., J. Pergner, I. Kozmiková, P. Fabian, A. R. Pombinho, H. Strnad, J. Pačes, Č. Vlček, P. Bartůněk, \& Z. Kozmik, 2015. Cubozoan genome illuminates functional diversification of opsins and photoreceptor evolution. Scientific Reports 5: 11885.

Lynch, M., \& A. Force, 1999. The Probability of Duplicate Gene Preservation by Subfunctionalization. Genetics 154: 459-473.

Marshall, J., K. L. Carleton, \& T. Cronin, 2015. Colour vision in marine organisms. Current Opinion in Neurobiology 34: 86-94.

Matsumoto, T., \& Y. Ishibashi, 2016. Sequence analysis and expression patterns of opsin genes in the longtooth grouper Epinephelus bruneus. Fisheries Science 82: $17-27$.

Menzel, R., 1979. Spectral Sensitivity and Color Vision in Invertebrates In Autrum, H. (ed), Comparative Physiology and Evolution of Vision in Invertebrates. Springer Berlin Heidelberg, Berlin, Heidelberg: 503-580.

Minh, B. Q., M. A. T. Nguyen, \& A. von Haeseler, 2013. Ultrafast Approximation for Phylogenetic Bootstrap. Molecular Biology and Evolution 30: 1188-1195.

Mirdita, M., L. von den Driesch, C. Galiez, M. J. Martin, J. Söding, \& M. Steinegger, 2017. Uniclust databases of clustered and deeply annotated protein sequences and alignments. Nucleic Acids Research 45: D170-D176.

Mirzadegan, T., G. Benkö, S. Filipek, \& K. Palczewski, 2003. Sequence Analyses of GProtein-Coupled Receptors: Similarities to Rhodopsin. Biochemistry 42: 27592767.

Morris, A., J. K. Bowmaker, \& D. M. Hunt, 1993. The molecular basis of a spectral shift in the rhodopsins of two species of squid from different photic environments. Proceedings of the Royal Society B: Biological Sciences 254: 233-240. 
Nathans, J., 1987. Molecular biology of visual pigments. Annual review of neuroscience 10: 163-194.

Nguyen, L.-T., H. A. Schmidt, A. von Haeseler, \& B. Q. Minh, 2015. IQ-TREE: A Fast and Effective Stochastic Algorithm for Estimating Maximum-Likelihood Phylogenies. Molecular Biology and Evolution 32: 268-274.

Nordström, K., T. A. Larsson, \& D. Larhammar, 2004. Extensive duplications of phototransduction genes in early vertebrate evolution correlate with block (chromosome) duplications. Genomics 83: 852-872.

Oakley, T. H., M. A. Alexandrou, R. Ngo, M. S. Pankey, C. K. Churchill, W. Chen, \& K. B. Lopker, 2014. Osiris: accessible and reproducible phylogenetic and phylogenomic analyses within the Galaxy workflow management system. BMC Bioinformatics 15: 230.

Oakley, T. H., \& D. R. Huber, 2004. Differential Expression of Duplicated Opsin Genes in Two EyeTypes of Ostracod Crustaceans. Journal of Molecular Evolution 59: 239-249.

Ohno, S., 1970. Evolution by gene duplication. George Allen and Unwin, London.

Panda, S., S. K. Nayak, B. Campo, J. R. Walker, J. B. Hogenesch, \& T. Jegla, 2005. Illumination of the Melanopsin Signaling Pathway. Science 307: 600-604.

Passamaneck, Y. J., N. Furchheim, A. Hejnol, M. Q. Martindale, \& C. Lüter, 2011. Ciliary photoreceptors in the cerebral eyes of a protostome larva. EvoDevo 2: 6.

Pearson, W. R., 2013. An Introduction to Sequence Similarity ("Homology") Searching In Baxevanis, A. D., G. A. Petsko, L. D. Stein, \& G. D. Stormo (eds), Current Protocols in Bioinformatics. John Wiley \& Sons, Inc., Hoboken, NJ, USA.

Pegueroles, C., S. Laurie, \& M. M. Albà, 2013. Accelerated Evolution after Gene Duplication: A Time-Dependent Process Affecting Just One Copy. Molecular Biology and Evolution 30: 1830-1842.

Porter, M. L., J. R. Blasic, M. J. Bok, E. G. Cameron, T. Pringle, T. W. Cronin, \& P. R. Robinson, 2012. Shedding new light on opsin evolution. Proceedings of the Royal Society B: Biological Sciences 279: 3-14.

Porter, M. L., M. J. Bok, P. R. Robinson, \& T. W. Cronin, 2009. Molecular diversity of visual pigments in Stomatopoda (Crustacea). Visual Neuroscience 26: 255-265.

Porter, M. L., T. W. Cronin, D. A. McClellan, \& K. A. Crandall, 2007. Molecular Characterization of Crustacean Visual Pigments and the Evolution of Pancrustacean Opsins. Molecular Biology and Evolution 24: 253-268. 
Porter, M. L., D. I. Speiser, A. K. Zaharoff, R. L. Caldwell, T. W. Cronin, \& T. H. Oakley, 2013. The Evolution of Complexity in the Visual Systems of Stomatopods: Insights from Transcriptomics. Integrative and Comparative Biology 53: 39-49.

Provencio, I., G. Jiang, W. J. De Grip, W. PÄR HAYES, \& M. D. Rollag, 1998. Melanopsin: An opsin in melanophores, brain, and eye. Proceedings of the National Academy of Sciences of the United States of America 95: 340-345.

Provencio, I., I. R. Rodriguez, G. Jiang, W. P. Hayes, E. F. Moreira, \& M. D. Rollag, 2000. A novel human opsin in the inner retina. Journal of Neuroscience 20: 600605.

Qiu, X., T. Kumbalasiri, S. M. Carlson, K. Y. Wong, V. Krishna, I. Provencio, \& D. M. Berson, 2005. Induction of photosensitivity by heterologous expression of melanopsin. Nature 433: 745-749.

Ramirez, M. D, A. N. Pairett, M. S. Pankey, J. M. Serb, D. I. Speiser, A. J. Swafford, T. H. Oakley, 2016. The Last Common Ancestor of Most Bilaterian Animals Possessed at Least Nine Opsins. Genome Biology and Evolution 8: 3640-3652.

Rasmussen, T. K., \& T. Krink, 2003. Improved Hidden Markov Model training for multiple sequence alignment by a particle swarm optimization-evolutionary algorithm hybrid. Biosystems 72: 5-17.

Remmert, M., A. Biegert, A. Hauser, \& J. Söding, 2012. HHblits: lightning-fast iterative protein sequence searching by HMM-HMM alignment. Nature Methods 9: 173175.

Sakamoto, K., O. Hisatomi, F. Tokunaga, \& E. Eguchi, 1996. Two opsins from the compound eye of the crab Hemigrapsus sanguineus. Journal of Experimental Biology 199: 441-450.

Shichida, Y., \& T. Matsuyama, 2009. Evolution of opsins and phototransduction. Philosophical Transactions of the Royal Society B: Biological Sciences 364: 2881-2895.

Simão, F. A., R. M. Waterhouse, P. Ioannidis, E. V. Kriventseva, \& E. M. Zdobnov, 2015. BUSCO: assessing genome assembly and annotation completeness with single-copy orthologs. Bioinformatics 31: 3210-3212.

Sjölander, K., 2004. Phylogenomic inference of protein molecular function: advances and challenges. Bioinformatics 20: 170-179.

Smith-Unna, R., C. Boursnell, R. Patro, J. M. Hibberd, \& S. Kelly, 2016. TransRate: reference-free quality assessment of de novo transcriptome assemblies. Genome Research 26: 1134-1144. 
Soubrier, J., M. Steel, M. S. Y. Lee, C. Der Sarkissian, S. Guindon, S. Y. W. Ho, \& A. Cooper, 2012. The Influence of Rate Heterogeneity among Sites on the Time Dependence of Molecular Rates. Molecular Biology and Evolution 29: 33453358.

Speiser, D. I., M. Pankey, A. K. Zaharoff, B. a Battelle, H. D. Bracken-Grissom, J. W. Breinholt, S. M. Bybee, T. W. Cronin, A. Garm, A. R. Lindgren, N. H. Patel, M. L. Porter, M. E. Protas, A. S. Rivera, J. M. Serb, K. S. Zigler, K. a Crandall, \& T. H. Oakley, 2014. Using phylogenetically-informed annotation (PIA) to search for light-interacting genes in transcriptomes from non-model organisms. BMC Bioinformatics 15: 350-350.

Stamatakis, A., 2014. RAxML version 8: a tool for phylogenetic analysis and postanalysis of large phylogenies. Bioinformatics 30: 1312-1313.

Stieb, S. M., F. Cortesi, L. Sueess, K. L. Carleton, W. Salzburger, \& N. J. Marshall, 2017. Why UV vision and red vision are important for damselfish (Pomacentridae): structural and expression variation in opsin genes. Molecular Ecology 26: 1323-1342.

Suzuki, S., T. Ishida, K. Kurokawa, \& Y. Akiyama, 2012. GHOSTM: A GPU-Accelerated Homology Search Tool for Metagenomics. PLoS ONE 7: e36060.

Terakita, A., 2005. The opsins. Genome biology 6: 213.

Tong, D., N. S. Rozas, T. H. Oakley, J. Mitchell, N. J. Colley, \& M. J. McFall-Ngai, 2009. Evidence for light perception in a bioluminescent organ. Proceedings of the National Academy of Sciences 106: 9836-9841.

Tsukamoto, H., I.-S. Chen, Y. Kubo, \& Y. Furutani, 2017. A ciliary opsin in the brain of a marine annelid zooplankton is ultraviolet-sensitive, and the sensitivity is tuned by a single amino acid residue. Journal of Biological Chemistry 292: 12971-12980.

Tutar, Y., 2012. Pseudogenes. Comparative and Functional Genomics 2012: 1-4.

Waterhouse, R. M., F. Tegenfeldt, J. Li, E. M. Zdobnov, \& E. V. Kriventseva, 2013. OrthoDB: a hierarchical catalog of animal, fungal and bacterial orthologs. Nucleic Acids Research 41: D358-D365.

Wen, Y.-Z., L.-L. Zheng, L.-H. Qu, F. J. Ayala, \& Z.-R. Lun, 2012. Pseudogenes are not pseudo any more. RNA Biology 9: 27-32.

Wong, J. M., J. L. Pérez-Moreno, T.-Y. Chan, T. M. Frank, \& H. D. Bracken-Grissom, 2015. Phylogenetic and transcriptomic analyses reveal the evolution of bioluminescence and light detection in marine deep-sea shrimps of the family Oplophoridae (Crustacea: Decapoda). Molecular Phylogenetics and Evolution 83: 278-292. 
803 Xu, P., R. Feuda, B. Lu, H. Xiao, R. I. Graham, \& K. Wu, 2016. Functional opsin $804 \quad$ retrogene in nocturnal moth. Mobile DNA 7: 18.

805 Yamada, K. D., K. Tomii, \& K. Katoh, 2016. Application of the MAFFT sequence 806 alignment program to large data-reexamination of the usefulness of chained 807 guide trees. Bioinformatics 32: 3246-3251.

808 Yoon, B.-J., 2009. Hidden Markov models and their applications in biological sequence 809 analysis. Current genomics 10: 402-415.

810 Zhang, J., 2003. Evolution by gene duplication: an update. Trends in Ecology \& 811 Evolution 18: 292-298.

812 Zhou, X., X.-X. Shen, C. T. Hittinger, \& A. Rokas, 2017. Evaluating Fast Maximum

813 Likelihood-Based Phylogenetic Programs Using Empirical Phylogenomic Data $814 \quad$ Sets. bioRxiv 142323.

815 


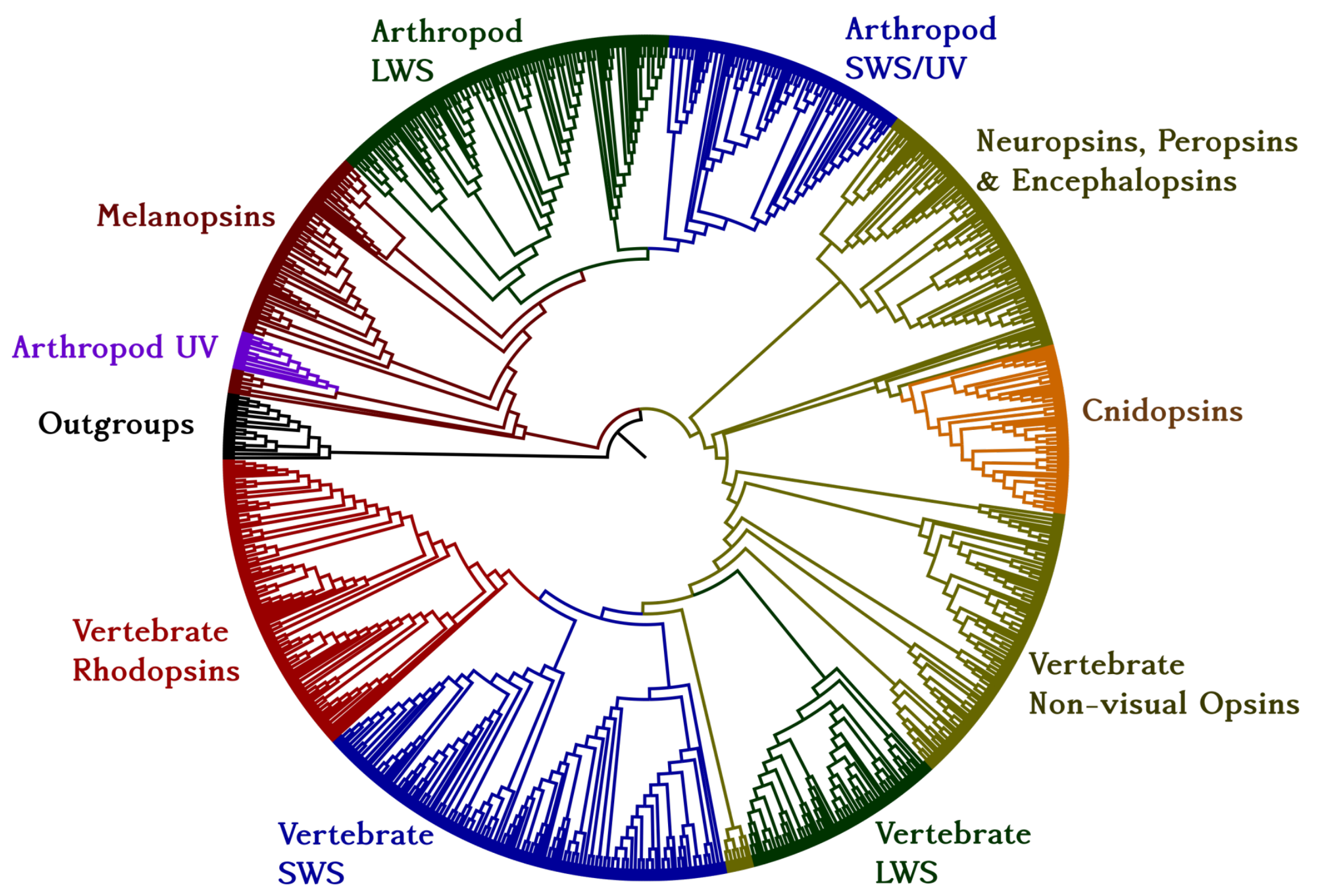

Figure 1. Maximum-Likelihood phylogeny of opsins estimated using putative opsin proteins identified by our annotation pipeline from the de novo transcriptome assemblies of Hyalella azteca and Daphnia pulex, along with a dataset of reference opsin sequences. Clades are annotated with opsin types contained therein and, in the case of visual opsins, with their inferred spectral sensitivities. 


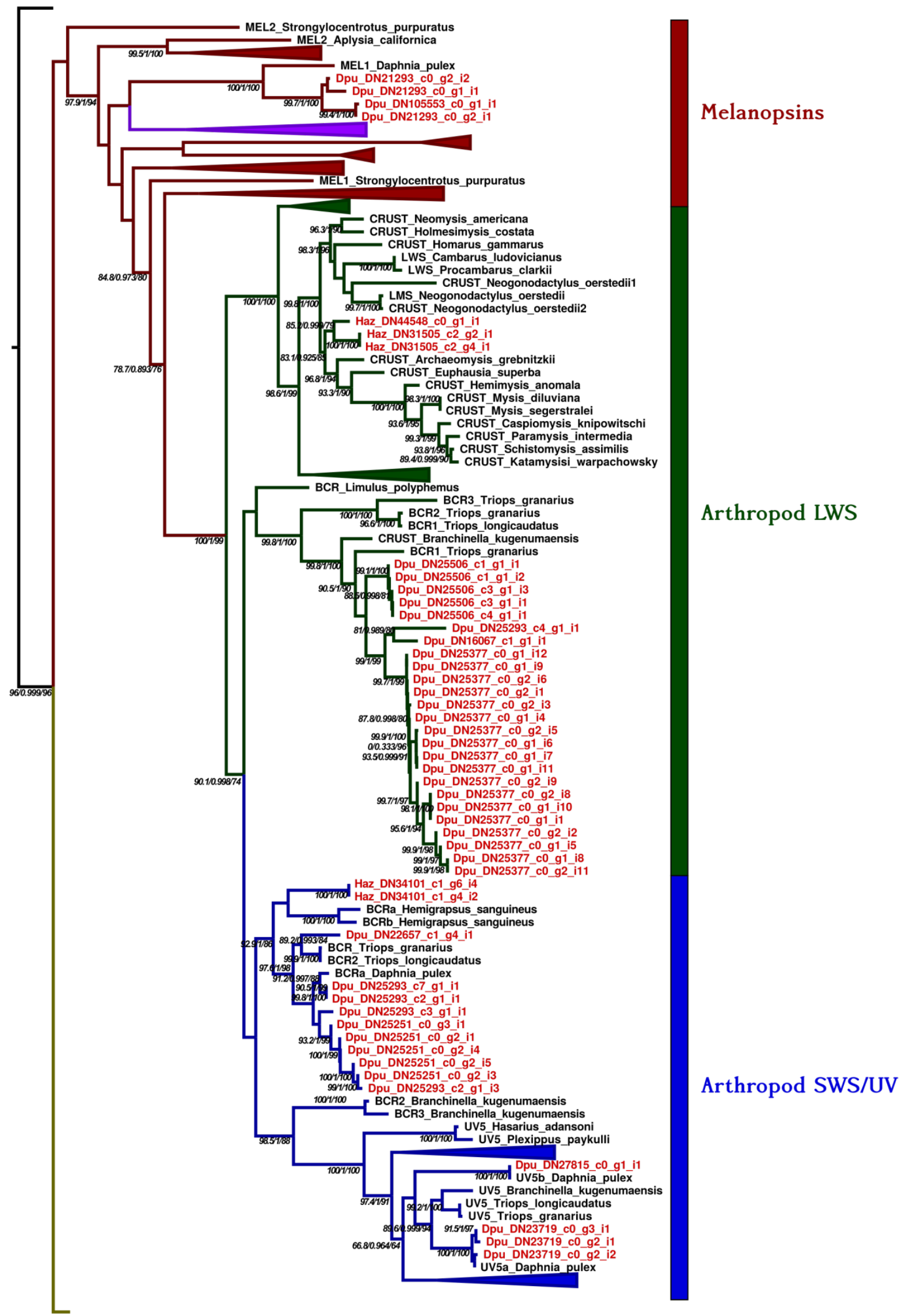

Figure 2. Expanded view of the melanopsin, Arthropod LWS, and Arthropod SWS/UV clades. Large non-crustacean clades have been collapsed for readability. Support values correspond to SH-aLRT/aBayes/UFBoot., and are not shown when bootstrap support $<75$. 


\section{Hyalella axteca}

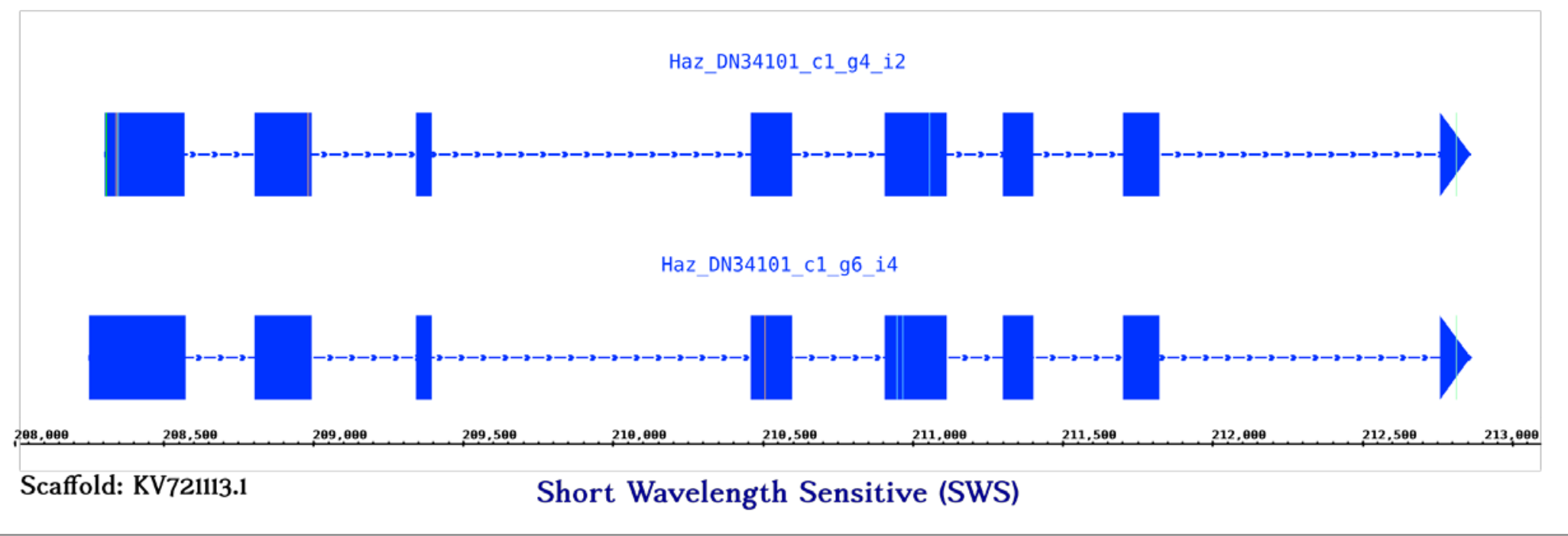

Haz_DN31505_c2_g4_i1

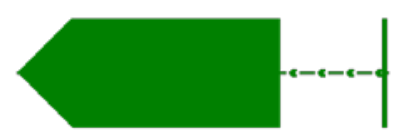

Haz_DN31505_c2_g2_i1

Haz_DN44548_c0_g1_il

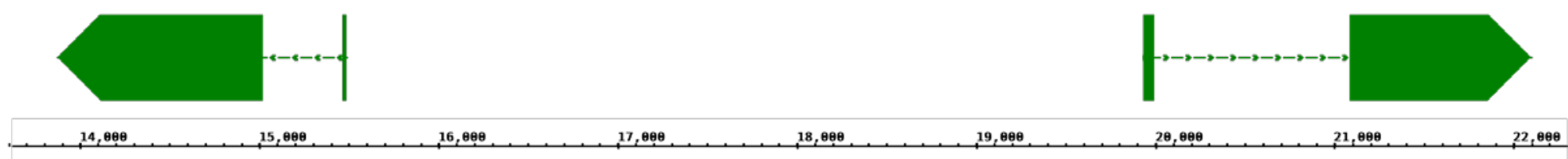

Scaffold: JQDR02013156.1

Long Wavelength Sensitive (SWS)

2 Figure 3. Intron-Exon gene structure patterns of representative Hyalella azteca SWS/UV and LWS opsins. A SAM alignment file with all of the mapped

3 transcripts is provided in the fighshare respository: http://figshare.com/xxxxx. 


\section{Daphnia pulex}

Dpu_DN22657_c1_g4_il

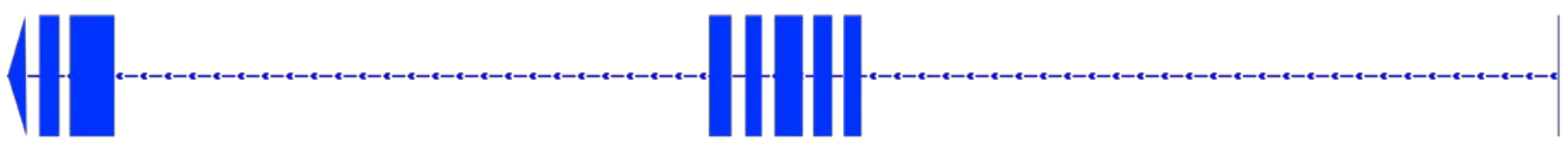

Dpu_DN22657_c1_g4_i1

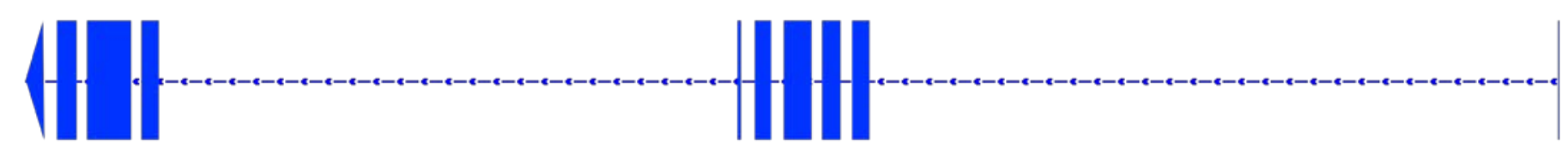

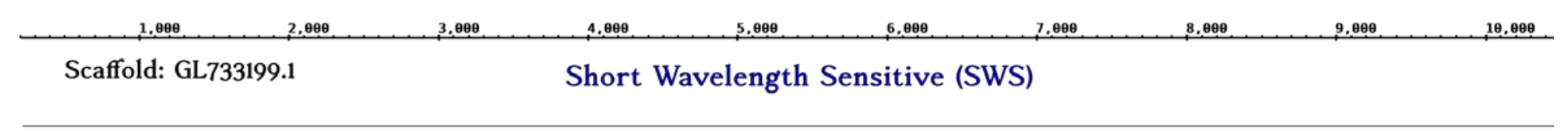

Dpu_DN25506_c3_g1_i1

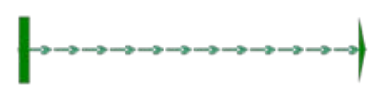

Dpu_DN25506_c1_g1_i2

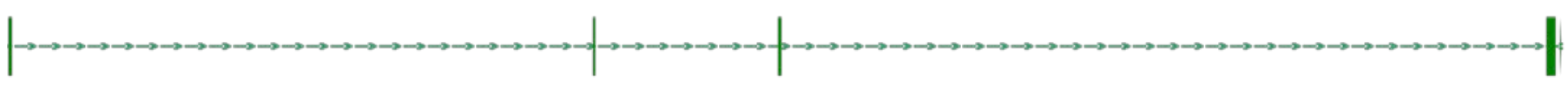

Dpu_DN25506_c4_g1_i1

III

,110,090. Long Wavelength Sensitive (SWS)

Dpu_DN25506_c3_g1_i3

Scaffold: GL732600.

Figure 4. Intron-Exon gene structure patterns of representative Daphnia pulex opsins SWS/UV and LWS opsins, which are arranged in the genome in distinct 


\section{Exon size distribution}

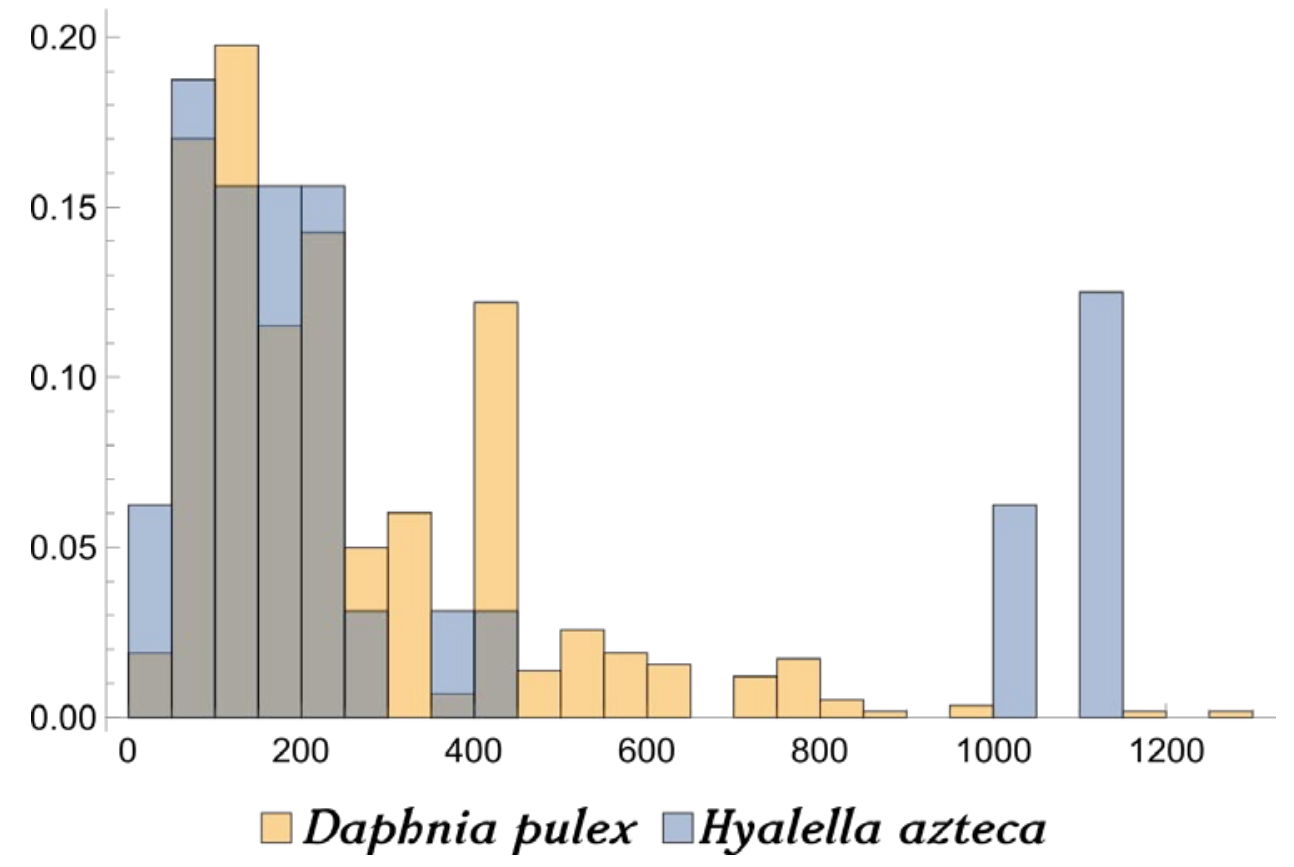

7 Figure 5. Exon size distribution for both Daphnia pulex and Hyalella azteca. X-axis is in base pair units (bp), while Y-axis represents proportion of transcripts in 8 said range. 